\title{
PD-L1 expression in non-small cell lung cancer: heterogeneity by pathologic types, tissue sampling and metastasis
}

\author{
Xuxia Shen ${ }^{1,2,3 \#}$, Yue Wang ${ }^{1,2,3 \#} \wedge$, Yan Jin ${ }^{1,2,3}$, Qiang Zheng ${ }^{1,2,3}$, Lei Shen ${ }^{1,2,3}$, Ying Chen ${ }^{1,2,3}$, Yuan Li ${ }^{1,2,3}$ \\ ${ }^{1}$ Department of Pathology, Fudan University Shanghai Cancer Center, Shanghai, China; ${ }^{2}$ Department of Oncology, Shanghai Medical College, Fudan \\ University, Shanghai, China; ${ }^{3}$ Shanghai Engineering Research Center of Artificial Intelligence Technology for Tumor Diseases, Shanghai, China \\ Contributions: (I) Conception and design: Y Li, Y Chen; (II) Administrative support: Y Li, L Shen; (III) Provision of study materials or patients: Y \\ Jin, Q Zheng; (IV) Collection and assembly of data: X Shen, Y Wang, Y Jin; (V) Data analysis and interpretation: X Shen, Y Wang; (VI) Manuscript \\ writing: All authors; (VII) Final approval of manuscript: All authors. \\ \#These authors contributed equally to this work. \\ Correspondence to: Yuan Li, MD, PhD. Department of Pathology, Fudan University Shanghai Cancer Center, Shanghai 200032, China; Department \\ of Oncology, Shanghai Medical College of Fudan University, Shanghai 200032, China. Email: whliyuan@hotmail.com; Ying Chen, MD, PhD. \\ Department of Pathology, Fudan University Shanghai Cancer Center, Shanghai 200032, China; Department of Oncology, Shanghai Medical College \\ of Fudan University, Shanghai 200032, China. Email: yingwxd@163.com.
}

Background: Programmed cell death ligand-1 (PD-L1) is a predictive marker of anti-PD-1/PD-L1 therapy response. Intra-tumour heterogeneity of PD-L1 expression has been reported in non-small cell lung cancer (NSCLC), but comprehensive studies regarding the determination of PD-L1 expression in different materials are still lacking. Therefore, we aimed to compare PD-L1 expression in paired tumour samples and in different specimen types.

Methods: A total of 1,002 resected NSCLC specimens, 35 biopsy specimens and 54 endobronchial ultrasound-guided transbronchial needle aspiration (EBUS-TBNA) samples were performed PD-L1 immunohistochemistry (IHC) testing using the 22C3 assay. PD-L1 expression was evaluated using the tumour proportion score (TPS) and categorized into three levels: negative (TPS $<1 \%$ ), low expression (TPS $1-49 \%$ ) and high expression (TPS $\geq 50 \%$ ).

Results: A total of 1,002 resected NSCLC specimens, including 852 adenocarcinomas (ADCs) and 150 squamous cell carcinomas (SCCs); 35 paired biopsy and resected samples; 54 paired cell block and biopsy samples; 53 paired blocks from the same resected tissue and 49 paired primary and metastatic lesion samples were included in this study. Interestingly, high PD-L1 expression was significantly more frequent in poorly differentiated subtypes than in well-differentiated subtypes in the ADC subgroup $(\mathrm{P}<0.001)$. In the SCC subgroup, high PD-L1 expression was significantly more associated with the nonkeratinizing type than the keratinizing type $(\mathrm{P}=0.001)$. PD-L1 expression differed between cell blocks and matched biopsy specimens (discordance rate $=11.1 \%, 6 / 54$ ) and between biopsy and matched resected specimens (discordance rate $=31.4 \%, 11 / 35)$. PD-L1 expression differed between different paraffin blocks from the same resected specimen (discordance rate $=35.8 \%, 19 / 53$ ), and the discordance rate of PD-L1 expression between primary tumours and matched lymph node metastases was $28.6 \%(14 / 49)$.

Conclusions: Discordant PD-L1 expression is not uncommon in NSCLC and warrants additional studies and serious consideration when interpreting PD-L1 test results. Initial negative test results may lead to repeat PD-L1 testing in additional samples or the use of a different clone if necessary.

Keywords: Non-small cell lung cancer (NSCLC); PD-L1; immunohistochemistry (IHC)

Submitted Mar 08, 2021. Accepted for publication Jun 18, 2021.

doi: $10.21037 /$ jtd-21-388

View this article at: https://dx.doi.org/10.21037/jtd-21-388

^ ORCID: Xuxia Shen, 0000-0003-2302-7188; Yue Wang, 0000-0001-5054-9286. 


\section{Introduction}

Immunotherapy has rapidly developed for treatment of lung cancer. Immune checkpoint inhibitors, specifically, antiprogrammed cell death 1/programmed cell death ligand 1 (anti-PD-1/PD-L1) therapies, have shown anti-tumour effects with marked responses in patients with non-small cell lung cancer (NSCLC) (1-3). Some immune checkpoint inhibitors (pembrolizumab, nivolumab and atezolizumab) have been recommended as standard treatments according to the therapeutic algorithm included in the current guidelines for lung cancer treatment (4-6). For each immune checkpoint inhibitor, PD-L1 expression is assessed by using different PD-L1 immunohistochemistry (IHC) assays. The PD-L1 IHC assay has become a companion diagnostic test (7-9). In certain circumstances, the predictive value of $\mathrm{PD}-\mathrm{L} 1$ as a biomarker has been questioned due to observations of an anti-tumour response in patients with no evidence of PD-L1 expression. One explanation for this observation could be related to the variability of the PD-L1 IHC assay. Another explanation is that PD-L1 staining may show heterogeneity in the same tumour or the same patient.

Heterogeneous PD-L1 expression has been reported in NSCLC and may affect clinical decisions regarding anti-PD-1/PD-L1 therapy when clinicians are faced with discrepant biomarker results (10). Heterogeneity raises concern regarding whether PD-L1 expression in one specimen is representative of its expression elsewhere. Therefore, sampling error may lead to misclassification of PD-L1 expression status. Some studies have reported on PD-L1 staining regarding intra-tumour heterogeneity in the whole section and small biopsy specimens or intertumour heterogeneity between primary and metastatic tumours (11-15). However, comprehensive studies on the determination of PD-L1 expression between different materials are still lacking.

In this study, we comprehensively analysed intertumour and intra-tumour heterogeneity to optimize tumour sampling and improve its accuracy. We investigated discordant PD-L1 expression between paired paraffin blocks from the same surgical resection samples, between paired cell block and biopsy samples, between paired biopsy and resected specimens and between paired primary and lymph node metastatic lesions. We also evaluated correlations between PD-L1 expression and histopathological characteristics.

We present the following article in accordance with the STROBE reporting checklist (available at https://dx.doi. org/10.21037/jtd-21-388).

\section{Methods}

\section{Patient sample selection}

A retrospective review of the patient database was performed from January 2018 through February 2019 at the Fudan University Shanghai Cancer Center to enroll NSCLC patients who underwent surgical resection and PD-L1 IHC testing. Patients who received neoadjuvant chemotherapy were excluded. Large cell neuroendocrine carcinoma, adenosquamous carcinoma, large cell carcinoma and sarcomatoid carcinoma were excluded. In total, 1,002 NSCLC patients were selected for this study. Of the 1,002 patients with surgically resected NSCLC, 35 patients underwent a preoperative diagnostic biopsy via flexible bronchoscopy or computed tomography (CT)-guided core needle biopsy. A total of 49 paired primary and metastatic lymph node tumour samples and 53 paired paraffin blocks from the same resected samples were randomly selected from the 1,002 patients with resected NSCLC. In addition, a total of 54 paired cell blocks and histology paraffin blocks from the same EBUS-TBNA samples were selected for this study. The patients' clinicopathological characteristics are described in Table S1. Our study was conducted in accordance with the Declaration of Helsinki (as revised in 2013) and approved by the Ethics Committee of Fudan University Shanghai Cancer Center (file number 050432-4$1805 \mathrm{C}$ ), and informed consent was taken from all the patients.

\section{Histologic and cytologic sample processing and PD-L1 IHC testing}

Cell blocks were prepared using EBUS-TBNA samples in phosphate-buffered saline. Effusion samples were centrifuged, the supernatant fluid was removed, and the precipitate was collected. The precipitate was further fixed in $10 \%$ neutral buffered formalin for at least 6 hours, embedded in paraffin, and sliced into $4-\mu \mathrm{m}$ sections for haematoxylin-eosin (HE) and PD-L1 IHC staining. Each sample was blindly assessed by two pathologists. Both histologic and cytologic diagnoses were classified according to the 2015 World Health Organization classification (16). Cases with less than one hundred viable tumour cells were excluded. PD-L1 IHC staining was performed on 4- $\mu \mathrm{m}$ thick formalin-fixed paraffin-embedded (FFPE) sections 
using the Dako PD-L1 IHC 22C3 pharmDx assay (Dako/ Agilent, Santa Clara, USA) on the Dako Link 48 automated platform according to the manufacturer's protocol. To avoid PD-L1 expression being influenced by the storage time of FFPE tissue blocks, the specimens for PD-L1 testing were all detected immediately after excision or biopsy. PDL1 staining was evaluated using the tumour proportion score (TPS), which is defined as the percentage of viable tumour cells (at least one hundred) with complete or partial membrane staining and classified into three levels: negative (TPS $<1 \%$ ), low expression (TPS $1-49 \%$ ) and high expression (TPS $\geq 50 \%$ ). Then, we analysed the associations of PD-L1 expression with histopathological characteristics and assessed the discordance rate of the TPS between paired cell block and biopsy samples $(n=54)$, paired biopsy and resected samples $(n=35)$, paired paraffin blocks from the same resected samples $(\mathrm{n}=53)$ and paired primary and metastatic lymph node tumours $(n=49)$.

\section{Statistical analysis}

Statistical analyses were performed using the SPSS software system (version 24.0, Chicago, IL, USA). Chi-square or Fisher's exact tests and logistic regression analysis were used to determine if any association was evident between PD-L1 expression and histological characteristics. Kappa tests were used to analyse the concordance between paired specimens, and the strength of concordance was categorized as follows: kappa value $>0.75$, good agreement; 0.4 to 0.75 , moderate agreement; $<0.4$, poor agreement. All statistical values were determined using two-tailed statistical analyses, and a $P$ value $<0.05$ was considered to indicate a statistically significant difference.

\section{Results}

\section{PD-L1 expression beterogeneity in NSCLC surgical resection specimens}

Of the 1,002 resected NSCLC specimens, including 852 ADCs and 150 SCCs, 305 cases (30.4\%) had positive PDL1 expression, including 102 (10.2\%) with high expression and $203(20.3 \%)$ with low expression. Of the 852 ADCs, $211(24.8 \%)$ were PD-L1 positive, including 61 (7.2\%) with high expression and $150(17.6 \%)$ with low expression. Of the 150 SCCs, 94 (62.7\%) were PD-L1 positive, including $41(27.3 \%)$ with high expression and 53 (35.3\%) with low expression. High PD-L1 expression was significantly more common in SCCs than in ADCs (27.3\% vs. $7.2 \%$, $\mathrm{P}<0.001)$. In the ADC subgroup, high PD-L1 expression was observed in $0 \%(0 / 72)$ of lepidic, $2.2 \%(9 / 418)$ of acinar, $2.0 \%$ (4/196) of papillary, $29.7 \%$ (41/138) of solid and $25.0 \%(7 / 28)$ of micropapillary predominant variants. High PD-L1 expression was significantly more frequent in poorly differentiated histological variants, such as solid and micropapillary predominant variants, than in welldifferentiated variants, such as papillary, acinar and lepidic predominant variants $(\mathrm{P}<0.001)$ (Figure $1 A, B)$. Furthermore, logistic regression analysis showed that the solid and micropapillary subtypes (poorly differentiated variants) had significantly more frequent high PD-L1 expression than lepidic, acinar and papillary subtypes (well differentiated variants) $(\mathrm{P}<0.01)$ (Table $\mathrm{S} 2)$. In the SCC subgroup, high PD-L1 expression, low PD-L1 expression and negative were observed in $4.8 \%(1 / 21), 23.8 \%(5 / 21)$, and $71.4 \%$ $(15 / 21)$ of keratinizing types and in $31.0 \%$ (40/129), $37.2 \%$ (48/129), and $31.8 \%(41 / 129)$ of nonkeratinizing types, respectively. High PD-L1 expression was significantly associated with the nonkeratinizing type $(\mathrm{P}=0.001)$ (Table 1).

\section{Discordant expression between paired cytology and bistology specimens}

A total of 54 paired cell block and biopsy samples, including $42 \mathrm{ADCs}$ and $12 \mathrm{SCCs}$, were included in this study. The concordance rate was $88.9 \%$ (48/54) between the paired cytology-histology specimens from the same EBUS-TBNA tissue, with a kappa value of 0.804 (good agreement, $\mathrm{P}<0.001)$. However, $11.1 \%(6 / 54)$ of the cases were discordant when comparing the paired samples (Figure 1C,D,E,F). The cell block overestimated or underestimated PD-L1 status in 7.4\% (4/54) and 3.7\% (2/54) of the matched biopsy specimens (Table 2). Among the 6 discrepant cases, $50 \%(3 / 6)$ of cases had a PD-L1 TPS ranging from $0 \%$ to $10 \%$, and the other $50 \%(3 / 6)$ had a TPS ranging from $40 \%$ to $60 \%$. Furthermore, when specimens were stratified by histologic type, the overall concordance rate was $90.5 \%$ (38/42) in ADCs and $83.3 \%$ $(10 / 12)$ in SCCs. The agreement of PD-L1 expression between paired cytology and histology specimens was slightly lower in SCCs than in ADCs.

\section{Discordant expression between paired biopsy and resected specimens}

A total of 35 paired biopsy and resected specimens, 

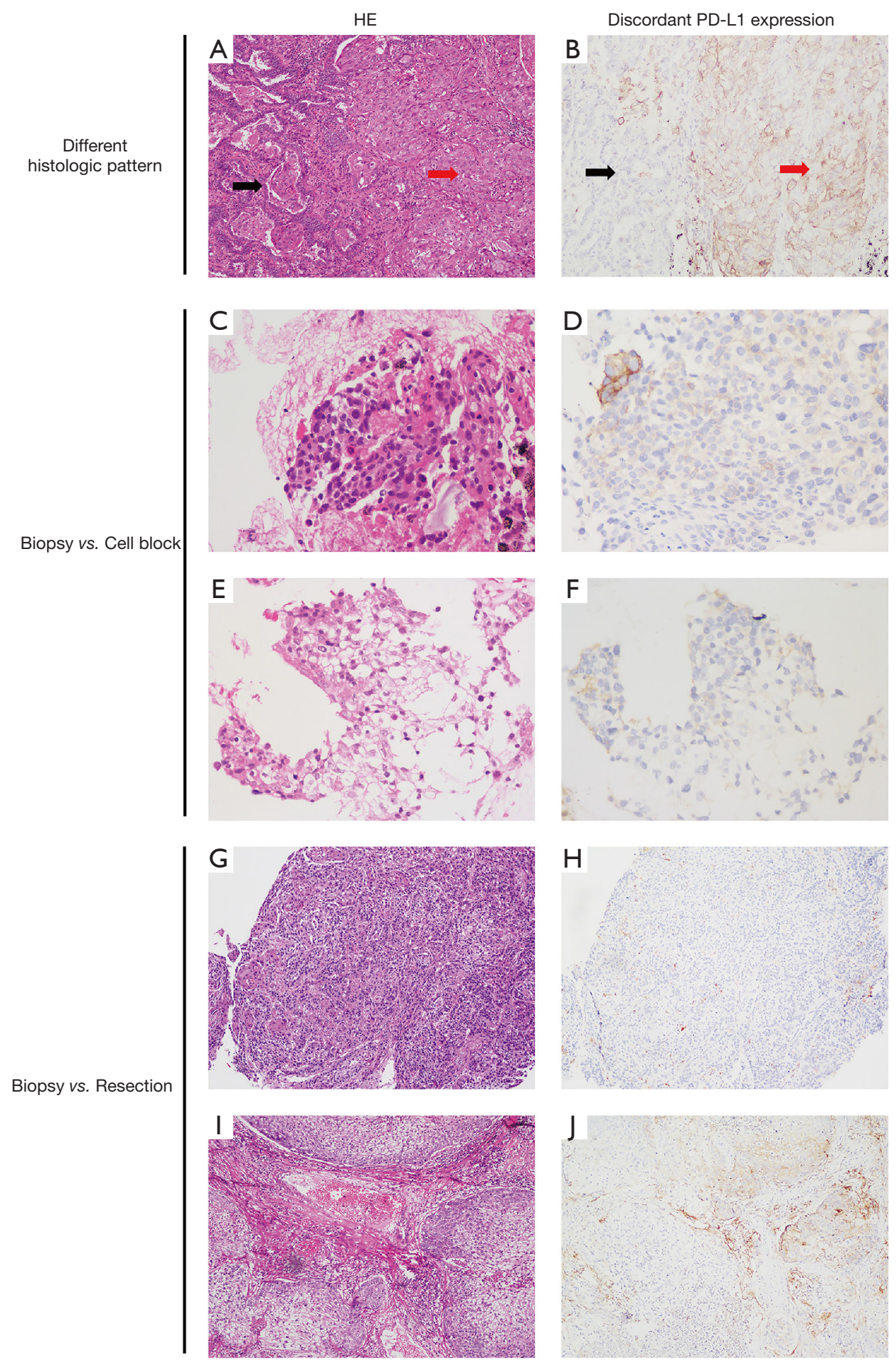

Figure 1 Discordant PD- L1 expression in NSCLC. (A,B) Discordant PD-L1 expression in different areas of the same adenocarcinoma: high expression in the solid area (red arrow) and negative in the acinar area (black arrow) (200×). (C,D,E,F) Discordant PD-L1 expression between paired biopsies and cell blocks from the same tumour: low expression in biopsies (C,D) and negative in cell blocks (E,F) (400x). $(\mathrm{G}, \mathrm{H}, \mathrm{I}, \mathrm{J})$ Discordant PD-L1 expression between paired biopsies and surgically resected specimens: negative in biopsies $(\mathrm{G}, \mathrm{H})$ and low expression in resected specimens $(\mathrm{I}, \mathrm{J})(100 \times)$. 
Table 1 The heterogeneous PD-L1 expression in different subtype and differentiation of ADC and SCC

\begin{tabular}{|c|c|c|c|c|c|c|}
\hline $\begin{array}{l}\text { Histologic } \\
\text { type }\end{array}$ & Subtype & \multicolumn{3}{|c|}{ PD-L1 status (\%) } & Total $(n=1,002)$ & $P$ value \\
\hline \multirow[t]{5}{*}{ ADC } & Subtype predominant & & & & & \\
\hline & Acinar & $351(84.0)$ & $58(13.9)$ & $9(2.2)$ & 418 & \\
\hline & Papillary & $160(81.6)$ & $32(16.3)$ & $4(2.0)$ & 196 & $<0.001$ \\
\hline & Solid & $51(37.0)$ & $46(33.3)$ & $41(29.7)$ & 138 & \\
\hline & Total & 641 & 150 & 61 & 852 & \\
\hline \multirow[t]{2}{*}{ SCC } & Keratinizing status & & & & & \\
\hline & Keratinizing & $15(71.4)$ & $5(23.8)$ & $1(4.8)$ & 21 & \\
\hline
\end{tabular}

PD-L1, programmed cell death ligand-1; ADC, adenocarcinoma; SCC, squamous cell carcinoma; TPS, tumour proportion score.

Table 2 The discrepant PD-L1 expression in the histology and matched cell block of the same biopsy sample

\begin{tabular}{|c|c|c|c|c|c|c|}
\hline \multirow{2}{*}{ PD-L1 status } & \multicolumn{3}{|c|}{ Cell blocks } & \multirow{2}{*}{ Total $(n=54)$} & \multirow{2}{*}{ Kappa value } & \multirow{2}{*}{$P$ value } \\
\hline & TPS $<1 \%$ & TPS 1-49\% & TPS $\geq 50 \%$ & & & \\
\hline \multicolumn{7}{|l|}{ Biopsy } \\
\hline TPS 1-49\% & 1 & 5 & 2 & 8 & & \\
\hline TPS $\geq 50 \%$ & 0 & 1 & 13 & 14 & & \\
\hline
\end{tabular}

PD-L1, programmed cell death ligand-1; TPS, tumour proportion score.

including 18 ADCs and 17 SCCs, were selected for this study. High PD-L1 expression was detected in $31.4 \%$ (11/35) of biopsy specimens and 28.6\% (10/35) of resected specimens, and low PD-L1 expression was detected in $28.6 \%(10 / 35)$ of biopsy specimens and $42.9 \%(15 / 35)$ of resected specimens. The overall concordance rate was $68.6 \%(24 / 35)$ (kappa value $=0.533$, moderate agreement, $\mathrm{P}<0.001)$. Between the paired biopsy and resected samples, a total of $31.4 \%(11 / 35)$ of the cases were found to be discordant (Figure 1G,H,I,7). The biopsy specimen overestimated and underestimated PD-L1 status in 11.4\% (4/35) and 20\% (7/35) of matched resected specimens, respectively (Table 3). Most discordant cases (8/11) had a PD-L1 TPS between $0 \%$ and $10 \%$. We also stratified the analysis according to histologic type. In ADCs and SCCs, the overall discordance rates were $16.7 \%(3 / 18)$ and $47.1 \%$
(8/17), respectively. The data showed that the concordance of PD-L1 expression between biopsy and resected specimens was lower in SCCs than in ADCs.

\section{Discordant expression in paired paraffin blocks from the same surgically resected samples}

A total of 53 paired paraffin blocks from the same surgically resected specimens (41 ADCs and 12 SCCs) were evaluated. The concordance rate of PD-L1 expression between the two paired blocks was $64.2 \%$ (34/53), with a kappa value of 0.455 (moderate agreement, $\mathrm{P}<0.001$ ). Discordant PD-L1 expression between the two blocks was detected in 35.8\% (19/53) of cases. Among them, $47.4 \%(9 / 19)$ and $21.1 \%$ (4/19) of cases had a PD-L1 TPS ranging from $0 \%$ to $10 \%$ and $40 \%$ to $60 \%$, respectively. We further stratified 
Table 3 The heterogeneous PD-L1 expression between biopsy and matched resected specimens

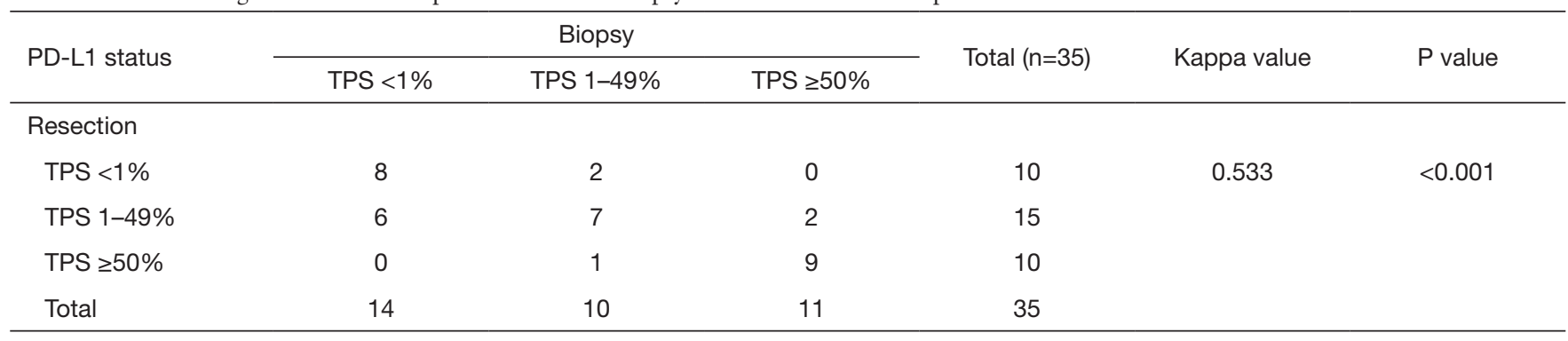

PD-L1, programmed cell death ligand-1; TPS, tumour proportion score.

Table 4 The discrepant PD-L1 expression between different paraffin blocks of the same surgically resected specimens

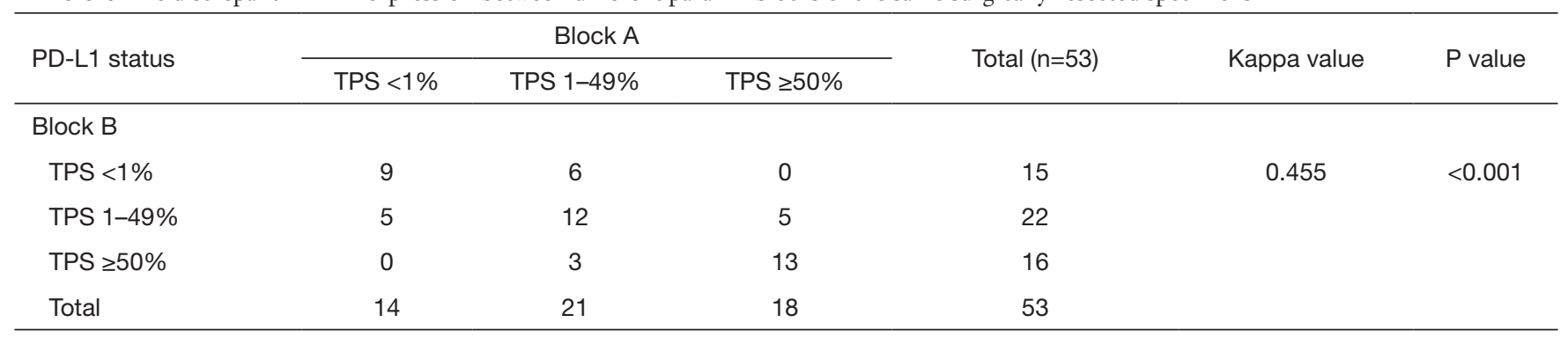

PD-L1, programmed cell death ligand-1; TPS, tumour proportion score.

the analysis according to histologic subtype and found that the overall disagreement rate was $29.3 \%(12 / 41)$ in ADCs and $58.3 \%(7 / 12)$ in SCCs. The concordance of PD-L1 expression between different blocks was lower in SCCs than in ADCs. In addition, among the 19 discordant cases, all 7 SCCs were nonkeratinizing types, 9 ADCs were solid and micropapillary predominant or minor variants, and $3 \mathrm{ADCs}$ were acinar and papillary predominant variants. In most ADCs (10/12) with discordant PD-L1 expression between the two blocks, each block had a different proportion of architectural growth patterns (Table 4, Figure 2).

\section{Discordant expression between paired primary tumour and metastatic lymph node lesion samples}

Of 49 paired primary and metastatic lymph node tumour samples, including $41 \mathrm{ADCs}$ and 8 SCCs, low and high PDL1 expression were observed in $36.7 \%(18 / 49)$ and $18.4 \%$ (9/49) of primary tumours and in $16.3 \%(8 / 49)$ and $24.5 \%$ $(12 / 49)$ of lymph node metastatic lesions, respectively. The concordance rate was $71.4 \%$ (35/49), with a kappa value of 0.546 (moderate agreement, $\mathrm{P}<0.001$ ). Between primary and matched metastatic lesions, a total of $28.6 \%(14 / 49)$ discordant cases were observed. The lymph node metastatic lesion overestimated or underestimated PD-L1 status in $10.2 \%(5 / 49)$ and $18.4 \%(9 / 49)$ of the matched primary tumours, respectively (Table 5). Among the 14 discrepant cases, $64.3 \%(9 / 14)$ of the cases had a PD-L1 TPS ranging from $0 \%$ to $10 \%$. Further stratified analysis was performed according to the histologic type. The overall discordance rate was 24.4\% (10/41) in ADCs and 50\% (4/8) in SCCs. The data showed that the concordance of PD-L1 expression between primary and metastatic lymph node tumours was lower in SCCs than in ADCs.

\section{Discussion}

PD-L1 expression is used as a predictive biomarker to select patients for PD-1/PD-L1 inhibitor therapy. Heterogeneous expression of $\mathrm{PD}-\mathrm{L} 1$ is pathophysiologically inducible and can be spatially and temporally variable and dynamic (11,17-19). Although there are published studies regarding heterogeneity, we comprehensively analysed the intertumour and intra-tumour PD-L1 expression in NSCLC. Using three-tiered categorization, our study showed that PD-L1 expression can be heterogeneous in subtypes of ADC, which was consistent with previous studies (18,20-23). High PD-L1 expression is significantly more frequent in 

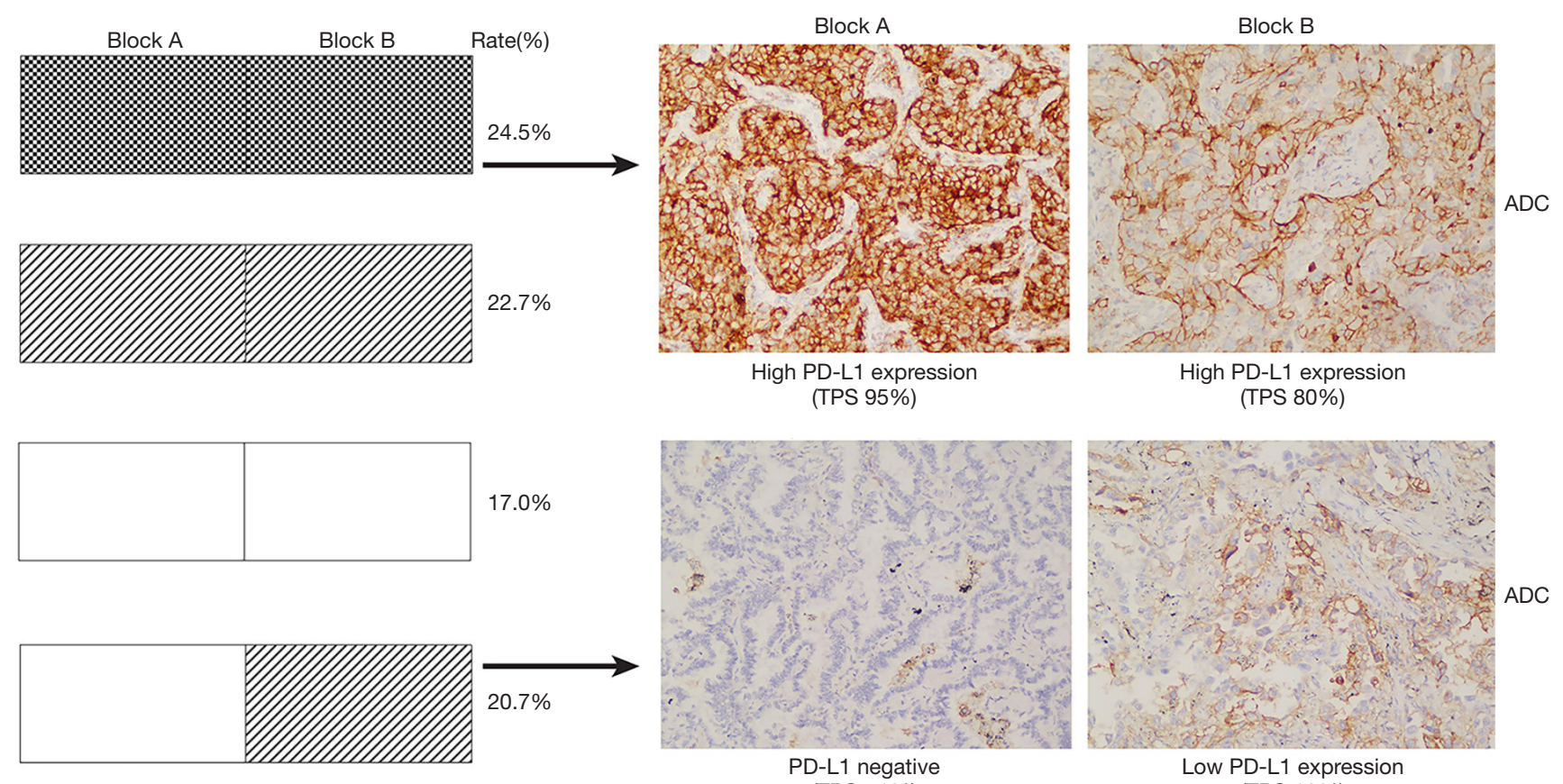

PD-L1 negative
(TPS $<1 \%$ )

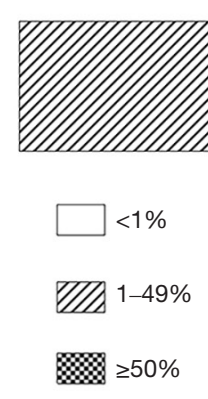

The concordance rate: $64.2 \%$ and the discordance rate: $35.8 \%$.
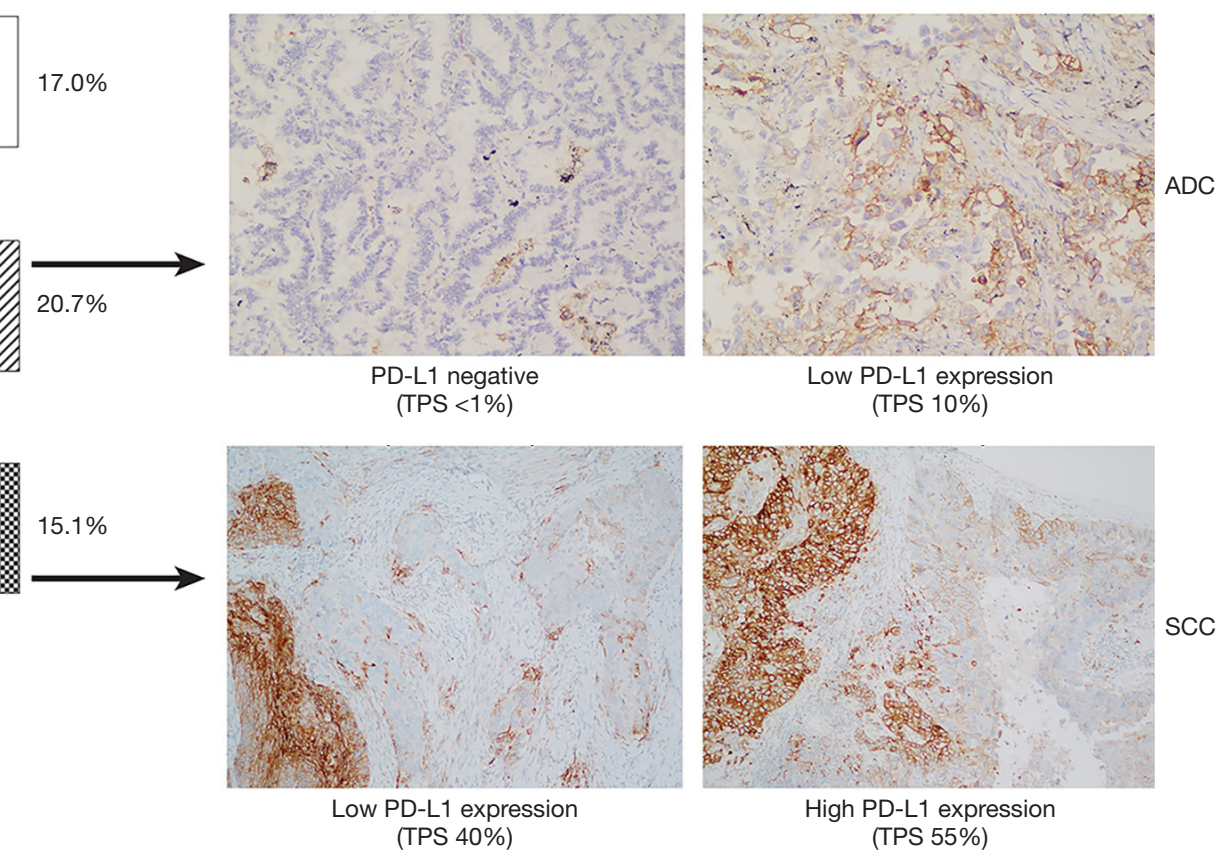

Figure 2 Heterogeneous expression of PD-L1 in different paraffin blocks of the same surgically resected sample. The left bar graph shows that the concordance and discordance rates of the two blocks were $64.2 \%$ and $35.8 \%$, respectively. Of the concordant cases (64.2\%), PD-L1 expression was high, low and negative in $24.5 \%, 22.7 \%$, and $17.0 \%$ of cases, respectively. The right IHC images show that the PD-L1 TPS in block A was $95 \%$ and in block B was $80 \%$ from the same tissue sample of ADC, both block A and B were high PD-L1 expression (200x). However, the discordant rate of two blocks was 35.8\%, including low expression $v$ s. negative (20.7\%) and high expression vs. low expression (15.1\%). The right IHC images show PD-L1 negative in block A and TPS 10\% (low expression) in block B from the same tissue sample of ADC (200×), and PD-L1 TPS 40\% (low expression) in block A and 55\% (high expression) in block B from the same tissue sample of SCC (100×). IHC staining. PD-L1, programmed cell death ligand-1; TPS, tumour proportion score; ADC, adenocarcinoma; SCC, squamous cell carcinoma.

poorly differentiated subtypes than in well-differentiated subtypes. Meanwhile, when using two-tiered categorization at a cut-off value of $50 \%$, the histologic subtypes of ADC were still significantly associated with $\mathrm{PD}-\mathrm{L} 1$ expression status $(\mathrm{P}<0.001$, see Table $\mathrm{S} 3)$. The histological subtypes of ADC may indicate PD-L1 expression status. The poorly differentiated subtypes (such as solid and micropapillary subtypes) might be more likely of high PD-L1 expression, while well-differentiated subtypes (lepidic, acinar and papillary subtypes) might be more likely of negative or low PD-L1 expression. This suggests that solid and micropapillary subtypes might activate PD-1/PD-L1 pathways, leading to suppression of anti-tumour immunity. However, in this study, the prevalence of PD-L1 expression 
Table 5 The heterogeneity of PD-L1 expression between primary and matched lymph node metastatic lesions

\begin{tabular}{|c|c|c|c|c|c|c|}
\hline PD-L1 status & \multicolumn{3}{|c|}{ Lymph node metastasis } & Total $(n=49)$ & Kappa value & $P$ value \\
\hline \multicolumn{7}{|l|}{ Primary lesion } \\
\hline TPS $<1 \%$ & 20 & 2 & 0 & 22 & 0.546 & $<0.001$ \\
\hline TPS $1-49 \%$ & 9 & 6 & 3 & 18 & & \\
\hline Total & 29 & 8 & 12 & 49 & & \\
\hline
\end{tabular}

PD-L1, programmed cell death ligand-1; TPS, tumour proportion score.

in $\mathrm{ADC}$ was relatively lower than that reported in previous studies. Previous studies have shown that PD-L1-positive expression was more frequent in advanced-stage and EGFR wild-type ADC $(20,24)$. The resected specimens in our cohort were mostly early-stage lung ADC with EGFR mutations, which may lead to a low $\mathrm{PD}-\mathrm{L} 1$ positivity rate. Furthermore, we identified that high PD-L1 expression is significantly associated with nonkeratinizing SCCs. Another significant and promising finding in our work was that discordant PD-L1 expression is more frequently observed in SCC than in ADC, as indicated by assessment of different intra-tumoural areas, paired tumour samples or different specimen types.

In advanced-stage NSCLC, physicians might obtain information from cytological samples, which are usually the only specimens available for diagnosis and biomarker testing. To date, PD-L1 expression has traditionally been evaluated in surgically excised samples, and the feasibility of using cytological samples for PD-L1 evaluation remains controversial. Recent studies evaluated PD-L1 expression in cell blocks and compared it with expression in paired biopsy or resection specimens. Using a three-tiered PD-L1 TPS categorization, the overall concordance rate between paired cytology and histology specimens was approximately 67 91.2\% (25-31). Our study provides some insights into PDL1 testing of paired biopsy and cytology specimens. PDL1 expression between the cell blocks and matched biopsy specimens had a high agreement rate of $88.9 \%$ (48/54) using three-tiered categorization, which is similar to the findings of previous reports. The PD-L1 TPS of discrepant cases mainly ranged from $0 \%$ to $10 \%$ and $40 \%$ to $60 \%$. These data indicate that cell blocks can be used in place of histology specimens to assess PD-L1 expression. These findings are important for clinicians as the cell blocks which are usually the only specimen available can be used for PDL1 expression testing.
Our study also compared PD-L1 expression in matched biopsy and resection samples from the same tumour and adds to the limited evidence of the feasibility of PDL1 testing in biopsy specimens. In a previous study, a major discordance rate of $48 \%$ in PD-L1 staining was reported between whole surgically resected specimens and matched biopsy specimens by using the SP142 assay, and these results have an impact on anti-PD-L1 therapeutic strategies (11). In our study, an agreement rate of $68.6 \%$ was found when comparing biopsy and paired resected samples by using the $22 \mathrm{C} 3$ assay, which was slightly lower than the agreement rate of $73-96 \%$ found in previous studies (32-34). Our data indicated that the PD-L1 status evaluated in a small biopsy is usually representative of the results in the resected specimen. However, the biopsy results could deviate from the PD-L1 expression status in resected specimens to a certain degree. A biopsy with high PD-L1 expression could be low expression in the resection specimen, or vice versa, and a biopsy with low PD-L1 expression could be negative in the resection specimen, or vice versa. The discordant PD-L1 expression between resection and biopsy samples could likely be attributed to intra-tumoural heterogeneity.

Assessment of intra-tumoural PD-L1 expression is a complicated process. Rehman et al. (35) observed that the heterogeneity between different blocks of the same tumour using the SP142 assay was not substantial. The variance in fields of view among each of the three blocks was only $6 \%$ for tumour areas but $25 \%$ for stromal areas. However, our data showed a $35.8 \%$ discrepancy in PD-L1 expression on tumour cells in different paraffin blocks using the $22 \mathrm{C} 3$ assay. Furthermore, our study showed that each block had a different proportion of architectural growth patterns in most discordant ADC cases. Our data and previous studies demonstrated that PD-L1 expression can be heterogeneous in different ADC subtypes. PD-L1 expression was more 
frequent in poorly differentiated subtypes, such as solid and micropapillary subtypes. Therefore, intra-tumoural histologic variance may play a role in discordant PD-L1 expression in different blocks, which was also confirmed by a previous study (23). More interestingly, in our study, $20.8 \%(11 / 53)$ of cases were negative in one block and positive in another block. These findings of discordant PD-L1 expression between different blocks highlight the importance of taking histological subtype into consideration when selecting a block for PD-L1 testing. If PD-L1 expression in one block is negative, adding an additional block for PD-L1 IHC testing may be useful to improve the PD-L1 positivity rate.

Metastatic lymph nodes are often used for diagnosis of advanced NSCLC patients. Previous studies have shown that the discrepancy in PD-L1 expression between the primary tumour and matched lymph node metastases in NSCLC is $32.3-71.4 \%(14,15,36)$. Our data showed a $28.6 \%$ discordance rate between the primary tumour and matched lymph node metastases, which was lower than that reported in previous studies. Several factors may influence PD-L1 expression, including the tumour hypoxia, the tumour microenvironment, and the activation of cellular pathways. We hypothesize that the different tumour cells from primary and metastatic lesions as well as their different tumour microenvironments can result in inconsistencies in PD-L1 expression.

In conclusion, we observed significantly discordant PD-L1 expression in different subtypes of ADCs and SCCs, between primary and metastatic lesions, between different paraffin blocks and between different specimen types. Specifically, PD-L1 expression in SCCs was more heterogeneous than that in ADCs. These discrepancies due to intra-tumoural or inter-tumoural heterogeneity may not be avoidable. This variability may pose a therapeutic dilemma in terms of whether immunotherapy should be recommended.

Our study is relatively comprehensive on assessing PD-L1 expression in different histological subtypes of NSCLC. But the limitation is that relatively small cohort of 4 paired samples were included in this study to explore the heterogeneity of NSCLC between different samples. We hope that more data will be available in future studies to further confirm our results. Secondly, our study was limited by the inherent issues of retrospective studies, including potential selection bias. This should be considered when generalizing our findings to other populations.

\section{Acknowledgments}

Funding: This study was supported by the National Nature Science Foundation of China [grant numbers 81972171 and 81472173].

\section{Footnote}

Reporting Checklist: The authors have completed the STROBE reporting checklist. Available at https://dx.doi. org/10.21037/jtd-21-388

Data Sharing Statement: Available at https://dx.doi. org/10.21037/jtd-21-388

Peer Review File: Available at https://dx.doi.org/10.21037/ jtd-21-388

Conflicts of Interest: All authors have completed the ICMJE uniform disclosure form (available at https://dx.doi. org/10.21037/jtd-21-388). The authors have no conflicts of interest to declare.

Ethical Statement: The authors are accountable for all aspects of the work and for ensuring that questions related to the accuracy or integrity of any part of the work are appropriately investigated and resolved. The study was conducted in accordance with the Declaration of Helsinki (as revised in 2013). The study was approved by the Ethics Committee of Fudan University Shanghai Cancer Center (file number 050432-4-1805C) and informed consent was taken from all the patients.

Open Access Statement: This is an Open Access article distributed in accordance with the Creative Commons Attribution-NonCommercial-NoDerivs 4.0 International License (CC BY-NC-ND 4.0), which permits the noncommercial replication and distribution of the article with the strict proviso that no changes or edits are made and the original work is properly cited (including links to both the formal publication through the relevant DOI and the license). See: https://creativecommons.org/licenses/by-nc-nd/4.0/.

\section{References}

1. Brahmer JR, Rodríguez-Abreu D, Robinson AG, et al. Health-related quality-of-life results for pembrolizumab versus chemotherapy in advanced, PD-L1-positive 
NSCLC (KEYNOTE-024): a multicentre, international, randomised, open-label phase 3 trial. Lancet Oncol 2017;18:1600-9.

2. Vokes EE, Ready N, Felip E, et al. Nivolumab versus docetaxel in previously treated advanced non-small-cell lung cancer (CheckMate 017 and CheckMate 057): 3-year update and outcomes in patients with liver metastases. Ann Oncol 2018;29:959-65.

3. Rittmeyer A, Barlesi F, Waterkamp D, et al. Atezolizumab versus docetaxel in patients with previously treated non-small-cell lung cancer (OAK): a phase 3, openlabel, multicentre randomised controlled trial. Lancet 2017;389:255-65.

4. Chen R, Tao Y, Xu X, et al. The efficacy and safety of nivolumab, pembrolizumab, and atezolizumab in treatment of advanced non-small cell lung cancer. Discov Med 2018;26:155-66.

5. Amrane K, Geier M, Corre R, et al. First-line pembrolizumab for non-small cell lung cancer patients with PD-L $1 \geq 50 \%$ in a multicenter real-life cohort: The PEMBREIZH study. Cancer Med 2020;9:2309-16.

6. Brahmer J, Reckamp KL, Baas P, et al. Nivolumab versus Docetaxel in Advanced Squamous-Cell Non-Small-Cell Lung Cancer. N Engl J Med 2015;373:123-35.

7. Büttner R, Gosney JR, Skov BG, et al. Programmed Death-Ligand 1 Immunohistochemistry Testing: A Review of Analytical Assays and Clinical Implementation in NonSmall-Cell Lung Cancer. J Clin Oncol 2017;35:3867-76.

8. Vennapusa B, Baker B, Kowanetz M, et al. Development of a PD-L1 Complementary Diagnostic Immunohistochemistry Assay (SP142) for Atezolizumab. Appl Immunohistochem Mol Morphol 2019;27:92-100.

9. Planchard D, Yokoi T, McCleod MJ, et al. A Phase III Study of Durvalumab (MEDI4736) With or Without Tremelimumab for Previously Treated Patients With Advanced NSCLC: Rationale and Protocol Design of the ARCTIC Study. Clin Lung Cancer 2016;17:232-6.e1.

10. Hirsch FR, McElhinny A, Stanforth D, et al. PDL1 Immunohistochemistry Assays for Lung Cancer: Results from Phase 1 of the Blueprint PD-L1 IHC Assay Comparison Project. J Thorac Oncol 2017;12:208-22.

11. Ilie M, Long-Mira E, Bence C, et al. Comparative study of the PD-L1 status between surgically resected specimens and matched biopsies of NSCLC patients reveal major discordances: a potential issue for anti-PD-L1 therapeutic strategies. Ann Oncol 2016;27:147-53.

12. Munari E, Zamboni G, Lunardi G, et al. PD-L1 Expression Heterogeneity in Non-Small Cell Lung
Cancer: Defining Criteria for Harmonization between Biopsy Specimens and Whole Sections. J Thorac Oncol 2018;13:1113-20.

13. Luo L, Luo X, Chen W, et al. Consistency Analysis of Programmed Death-Ligand 1 Expression between Primary and Metastatic Non-Small Cell Lung Cancer: A Retrospective Study. J Cancer 2020;11:974-82.

14. Xu H, Chen X, Lin D, et al. Conformance Assessment of PD-L1 Expression Between Primary Tumour and Nodal Metastases in Non-Small-Cell Lung Cancer. Onco Targets Ther 2019;12:11541-7.

15. Haragan A, Field JK, Davies MPA, et al. Heterogeneity of PD-L1 expression in non-small cell lung cancer: Implications for specimen sampling in predicting treatment response. Lung Cancer 2019;134:79-84.

16. Travis WD, Brambilla E, Burke AP, et al. WHO Classification of Tumours of the Lung, Pleura, Thymus and Heart. 4th ed. Lyon: IARC Press; 2015.

17. McLaughlin J, Han G, Schalper KA, et al. Quantitative Assessment of the Heterogeneity of PD-L1 Expression in Non-Small-Cell Lung Cancer. JAMA Oncol 2016;2:46-54.

18. Naso JR, Wang G, Pender A, et al. Intratumoral heterogeneity in programmed death-ligand 1 immunoreactivity is associated with variation in nonsmall cell lung carcinoma histotype. Histopathology 2020;76:394-403.

19. Naso JR, Banyi N, Al-Hashami Z, et al. Discordance in PD-L1 scores on repeat testing of non-small cell lung carcinomas. Cancer Treat Res Commun 2021;27:100353.

20. Song P, Wu S, Zhang L, et al. Correlation Between PDL1 Expression and Clinicopathologic Features in 404 Patients with Lung Adenocarcinoma. Interdiscip Sci 2019;11:258-65.

21. Lee SE, Kim YJ, Sung M, et al. Association with PD-L1 Expression and Clinicopathological Features in 1000 Lung Cancers: A Large Single-Institution Study of Surgically Resected Lung Cancers with a High Prevalence of EGFR Mutation. Int J Mol Sci 2019;20:4794.

22. Chen Q, Fu YY, Yue QN, et al. Distribution of PD-L1 expression and its relationship with clinicopathological variables: an audit from 1071 cases of surgically resected non-small cell lung cancer. Int J Clin Exp Pathol 2019;12:774-86.

23. Bulutay P, Firat P, Zeren EH, et al. The importance of histological patterns on PD-L1 staining heterogeneity: Should we use pattern-based approach for selecting tumor samples for PD-L1 testing in lung adenocarcinomas? Turk J Med Sci 2021;51:204-13. 
24. Jin Y, Shen X, Pan Y, et al. Correlation between PDL1 expression and clinicopathological characteristics of non-small cell lung cancer: A real-world study of a large Chinese cohort. J Thorac Dis 2019;11:4591-601.

25. Hernandez A, Brandler TC, Zhou F, et al. Assessment of Programmed Death-Ligand 1 (PD-L1) Immunohistochemical Expression on Cytology Specimens in Non-Small Cell Lung Carcinoma. Am J Clin Pathol 2019;151:403-15.

26. Kuempers C, van der Linde LIS, Reischl M, et al. Comparison of PD-L1 expression between paired cytologic and histologic specimens from non-small cell lung cancer patients. Virchows Arch 2020;476:261-71.

27. Noll B, Wang WL, Gong Y, et al. Programmed death ligand 1 testing in non-small cell lung carcinoma cytology cell block and aspirate smear preparations. Cancer Cytopathol 2018;126:342-52.

28. Grosu HB, Arriola A, Stewart J, et al. PD-L1 detection in histology specimens and matched pleural fluid cell blocks of patients with NSCLC. Respirology 2019;24:1198-203.

29. Jug R, Giovacchini CX, Liu B, et al. EBUSFNA cytologic-histologic correlation of PD-L1 immunohistochemistry in non-small cell lung cancer. J Am Soc Cytopathol 2020;9:485-93.

30. Chauhan A, Siegel L, Freese R, et al. Performance of Ventana SP263 PD-L1 assay in endobronchial ultrasound

Cite this article as: Shen X, Wang Y, Jin Y, Zheng Q, Shen L, Chen Y, Li Y. PD-L1 expression in non-small cell lung cancer: heterogeneity by pathologic types, tissue sampling and metastasis. J Thorac Dis 2021;13(7):4360-4370. doi: 10.21037/jtd21-388 guided-fine-needle aspiration derived non-small-cell lung carcinoma samples. Diagn Cytopathol 2021;49:355-62 .

31. Wang G, Ionescu DN, Lee CH, et al. PD-L1 testing on the EBUS-FNA cytology specimens of non-small cell lung cancer. Lung Cancer 2019;136:1-5.

32. Kim I, Kim A, Lee CH, et al. Reliability of PD-L1 assays using small tissue samples compared with surgical specimens. Medicine (Baltimore) 2019;98:e14972.

33. Elfving H, Mattsson JSM, Lindskog C, et al. Programmed Cell Death Ligand 1 Immunohistochemistry: A Concordance Study Between Surgical Specimen, Biopsy, and Tissue Microarray. Clin Lung Cancer 2019;20:258-62.e1.

34. Gradecki SE, Grange JS, Stelow EB. Concordance of PD-L1 Expression Between Core Biopsy and Resection Specimens of Non-Small Cell Lung Cancer. Am J Surg Pathol 2018;42:1090-4.

35. Rehman JA, Han G, Carvajal-Hausdorf DE, et al. Quantitative and pathologist-read comparison of the heterogeneity of programmed death-ligand 1 (PD-L1) expression in non-small cell lung cancer. Mod Pathol 2017;30:340-9.

36. Saito $\mathrm{Y}$, Horiuchi S, Morooka H, et al. Inter-tumor heterogeneity of PD-L1 expression in non-small cell lung cancer. J Thorac Dis 2019;11:4982-91. 
Supplementary

Table S1 Clinicopathological characteristics of NSCLC patients in the study

\begin{tabular}{|c|c|c|c|}
\hline \multirow[b]{2}{*}{ Variables } & \multirow[b]{2}{*}{ Resection ( $\mathrm{N}=1002)$} & \multicolumn{2}{|c|}{ Small biopsy } \\
\hline & & $\begin{array}{l}\text { Biopsy paired with resection } \\
\qquad(\mathrm{N}=35)\end{array}$ & EBUS-TBNA $(\mathrm{N}=54)$ \\
\hline \multicolumn{4}{|l|}{ Age (years) } \\
\hline Median (range) & $62(17-84)$ & $67(52-82)$ & $63.5(40-81)$ \\
\hline \multicolumn{4}{|l|}{ Sex } \\
\hline Male & 525 & 23 & 36 \\
\hline Female & 477 & 12 & 18 \\
\hline \multicolumn{4}{|l|}{ Smoking habits } \\
\hline Current/former & 386 & 18 & 33 \\
\hline Never & 616 & 17 & 21 \\
\hline \multicolumn{4}{|l|}{ Histology } \\
\hline Adenocarcinoma & 852 & 18 & 42 \\
\hline Squamous cell carcinoma & 150 & 17 & 12 \\
\hline \multicolumn{4}{|l|}{ UICC-stage } \\
\hline I & 799 & 11 & 1 \\
\hline ॥ & 115 & 5 & 1 \\
\hline III & 86 & 19 & 39 \\
\hline IV & 2 & 0 & 13 \\
\hline \multicolumn{4}{|l|}{ EGFR mutation status } \\
\hline Yes & 472 & 10 & 12 \\
\hline No & 154 & 8 & 13 \\
\hline Not tested & 376 & 17 & 29 \\
\hline \multicolumn{4}{|l|}{ Neoadjuvant therapy } \\
\hline Yes & 0 & 0 & 0 \\
\hline No & 1002 & 35 & 54 \\
\hline
\end{tabular}


Table S2 The Logistic Regression Analysis of PD-L1 expression in different subtypes of ADC (PD-L1 TPS cut-off value at 50\%)

\begin{tabular}{lccc}
\hline Subtype & OR & $95 \% \mathrm{Cl}$ & P value \\
\hline Lepidic vs. Micropapillary & 0.042 & $0.005-0.358$ & 0.004 \\
Lepidic vs. Solid & 0.034 & $0.012-0.100$ & $<0.001$ \\
Acinar vs. Micropapillary & 0.066 & $0.022-0.195$ & $<0.001$ \\
Acinar vs. Solid & 0.112 & $0.073-0.173$ & $<0.001$ \\
Papillary vs. Micropapillary & 0.063 & $0.017-0.231$ & $<0.001$ \\
Papillary vs. Solid & 0.132 & $0.080-0.218$ & $<0.001$ \\
Micropapillary vs. Solid & 0.906 & $0.394-2.085$ & 0.816 \\
\hline
\end{tabular}

Table S3 The heterogeneous PD-L1 expression in different subtype and differentiation of ADC (PD-L1 TPS cut-off value at $50 \%$ )

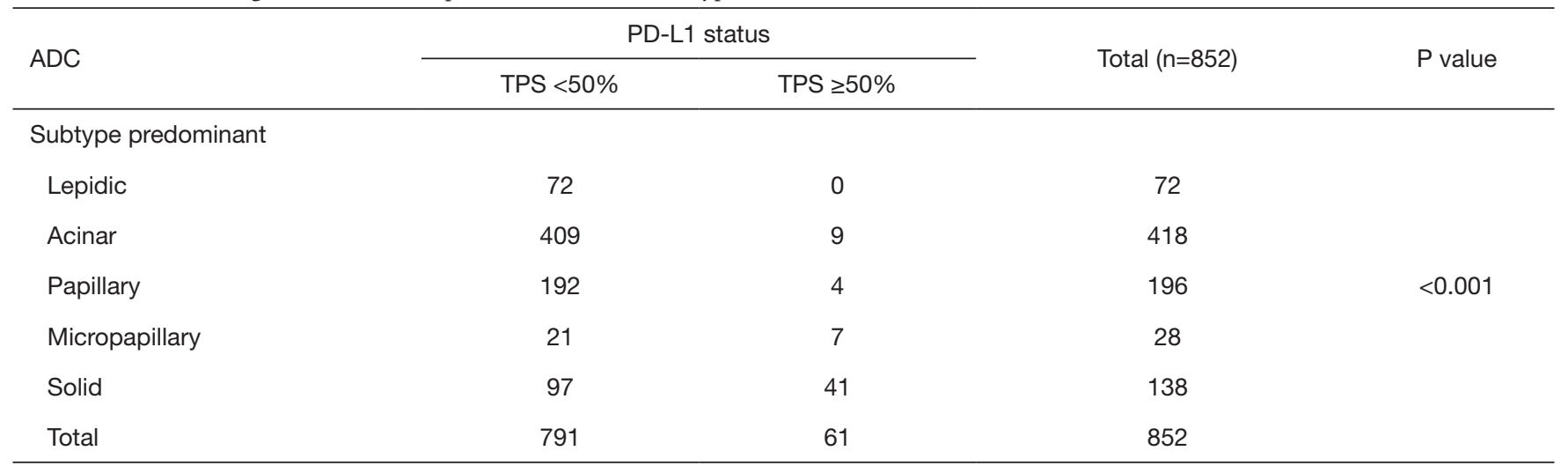

